\title{
Article \\ Research on the Modelling and Development of Flexibility in Production System Design Phase Driven by Digital Twins
}

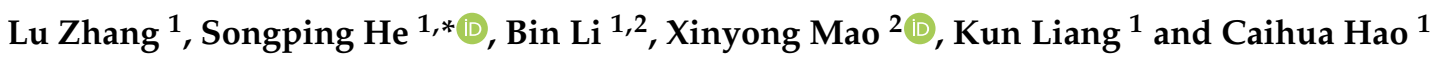 \\ 1 State Key Laboratory of Digital Manufacturing Equipment and Technology, Huazhong University of Science \\ and Technology, Wuhan 430074, China; zhanglu9189@hust.edu.cn (L.Z.); libin999@hust.edu.cn (B.L.); \\ liangkun1996@hust.edu.cn (K.L.); d202080306@hust.edu.cn (C.H.) \\ 2 National NC System Engineering Research Center, Huazhong University of Science and Technology, \\ Wuhan 430074, China; maoxyhust@hust.edu.cn \\ * Correspondence: hesongping@hust.edu.cn
}

check for

updates

Citation: Zhang, L.; He, S.; Li, B.;

Mao, X.; Liang, K.; Hao, C. Research on the Modelling and Development of Flexibility in Production System

Design Phase Driven by Digital

Twins. Appl. Sci. 2022, 12, 2537.

https://doi.org/10.3390/

app12052537

Academic Editor: Arcangelo

Castiglione

Received: 11 January 2022

Accepted: 22 February 2022

Published: 28 February 2022

Publisher's Note: MDPI stays neutral with regard to jurisdictional claims in published maps and institutional affiliations.

Copyright: (C) 2022 by the authors. Licensee MDPI, Basel, Switzerland. This article is an open access article distributed under the terms and conditions of the Creative Commons Attribution (CC BY) license (https:// creativecommons.org/licenses/by/ $4.0 /)$.

\begin{abstract}
Production system flexibility has become an important factor to meet the needs of diversified markets in an ever-changing and turbulent market environment. Thus, this paper addresses the problems of flexibility evaluation for decision making by evaluation method driven by digital twin, which models, predicts, and evaluates the flexibility of a given production system design scheme (PSDS). Based on the definition and characteristic analysis of flexibility in the production system design phase, and combined with data modelling (static function model (SFM) and dynamic prediction model (DPM)) and data analysis (AHP-based and simulation-based analysis), the proposed framework evaluates system flexibility from the static and dynamic levels, helps enterprises quickly obtain the system's comprehensive flexibility evaluation index, realizing the rapid screening of the production system design scheme (PSDS). The successful application of the evaluation framework in case enterprise has fully proved the feasibility and effectiveness of the method.
\end{abstract}

Keywords: digital twin; flexibility evaluation; digital model; flexibility definition and analysis

\section{Introduction}

Customized production systems have become an important means for enterprises to meet the current manufacturing market in facing the demand for multivariety and personalized products [1-3]. The custom development of the production system involves multiple design steps, such as demand analysis, schematic design, manufacturing, assembly, and commissioning, among which the schematic design is the most important link in the process of customized development. The customized design aims to obtain the optimal solution with the goal of meeting the production requirements. Therefore, the design process of the system scheme will be a long-term and iterative process, and many design schemes will be proposed for selection. This requires companies to have the ability to screen the optimal production system design scheme quickly and efficiently.

To meet the challenges of the current market, manufacturing flexibility has become the most important means for companies in designing production systems [4]. Flexibility plays an important role in the design, manufacture, and operation of production systems in enterprises, and it has been introduced into production systems for decades [5-10]. It helped BMW avoid layoffs in their U.S. plants in the face of the global financial crisis of 2008 and its negative impact on automotive sales [11]. Flexibility configuration capabilities are used by Nakajima et al. [12] for proposing a command-centric satellite software architecture which shortened the product development cycle and made partial satellite software reuse possible.

Flexibility [13] is an important technical means for manufacturing enterprises to transform from a traditional manufacturing mode to the new manufacturing mode, and is the driving force of technological innovation in the manufacturing industry. Jain et al. [14] proposed in a review study that manufacturing flexibility is a multidimensional concept, 
which exhibits different characteristics in different dimensions. Jain summarized 12 types of flexibility and made corresponding definitions for different types of flexibility. The flexibility types in the manufacturing industry summarized include machine flexibility, operation flexibility, path flexibility, volume flexibility, and so on. Each type of flexibility describes a certain aspect of the system. For example, machine flexibility describes the number and type of operations that the machine can perform. As formulas, algorithms, or methods, flexibility metrics can generate values for a given type of flexibility under a given condition.

To this end, researchers will evaluate the system flexibility to develop the system. Scholars' research on system flexibility will focus on different characteristics of flexibility according to their research purposes. As a multidimensional concept, flexibility has different characteristics and evaluation methods at different phases of the system. When the production system is in the schematic design phase, its flexibility is mainly reflected in the ability of the system to meet the production needs of the enterprise. The higher the ability of the system to meet the needs of the enterprise, the better the flexibility of the system designed. Thus, how to carry out the flexibility research of production systems in the design phase is the key to helping the system develop and meet the market demand.

Nowadays, with the development of the new generation of information and communication technologies, digital twin has achieved a breakthrough momentum of technological development [15-17]. Digital twin refers to the process and method of describing and modelling a physical object's characteristics, formation process, and performance using digital technology. It can simulate the behaviour and performance of the entities in real time based on information collection and sharing [18]. However, real-time data collection and information sharing cannot be realized when researching the production system in the design phase. The digital model [19] as the core component of the digital twin can help achieve object-oriented comprehensive information digitization expression and management to simulate the behaviour and performance of entities. Digital models include, but are not limited to, simulation models, mathematical models, etc., in which the data required to build the model can be exchanged with the physical model manually. The digital model realizes the analysis of system performance in the production system design phase (real-time data sharing cannot be automatically realized). It is also the basis for the construction of flexibility-oriented digital twin in the production and operation of the actual production system. Therefore, this paper studies the flexibility of a production system driven by digital twin technology.

However, driven by the digital twin technology, there are still many challenges in building a flexibility-oriented digital model for production systems, as well as to study system flexibility and help system development. First, how to build a flexibility research framework for the system driven by digital twin technology has become the primary issue. Transferring digital twin technology to applied research of system flexibility can help analyse system flexibility comprehensively. Secondly, the production system flexibility characteristics are related to the multidimensional information of the system, and the characteristics of the system flexibility show a high degree of complexity and coupling. Therefore, how to establish an accurate and comprehensive digital model of system flexibility has become a key issue. Thus, we needed an efficient and rapid research method to construct the flexibility digital model of the production system. With existing research challenges, the paper research framework is as follows. In Section 2, the flexibility research framework of the production system driven by digital twin technology in the design phase is introduced. Section 3, the construction of the flexibility-oriented digital model is studied. The paper combines the methods supported by the existing digital twin technology to find a suitable research method for system flexibility. Combined with real cases, this paper demonstrates the feasibility of the proposed modelling and research method for system flexibility in Section 4. Finally, in Section 5, a brief conclusion and ideas for further development are presented. 


\section{System Flexibility Framework Driven by Digital Twin}

As an effective means to realize the digital transformation and intelligent upgrading of traditional industries, digital twin technology can realize effective management and analysis of the entire product life cycle. Digital twin exists in the entire life cycle of the product, and uses virtual environments and simulation computing technologies to improve the performance of the system [20]. In the manufacturing industry, the ISO/DIS 23247-1 [21] standard defines that the digital twin can realize the digital expression of all aspects of observable objects, among which the observable objects can be personnel, equipment, materials, environment, supporting documents, etc. The supporting documents defined in this standard are any form of artefact (requirement, plan, model, specification, and configuration) that assists manufacturing. Based on the above foundation, with the support of digital twin technology, this paper proposes a flexibility research framework for production system in the design phase as shown in Figure 1. This framework is used to realize the data extraction, modelling and analysis of flexibility features in the production system design scheme, helping to make fast and accurate flexibility evaluation and optimization decision making for each design scheme.
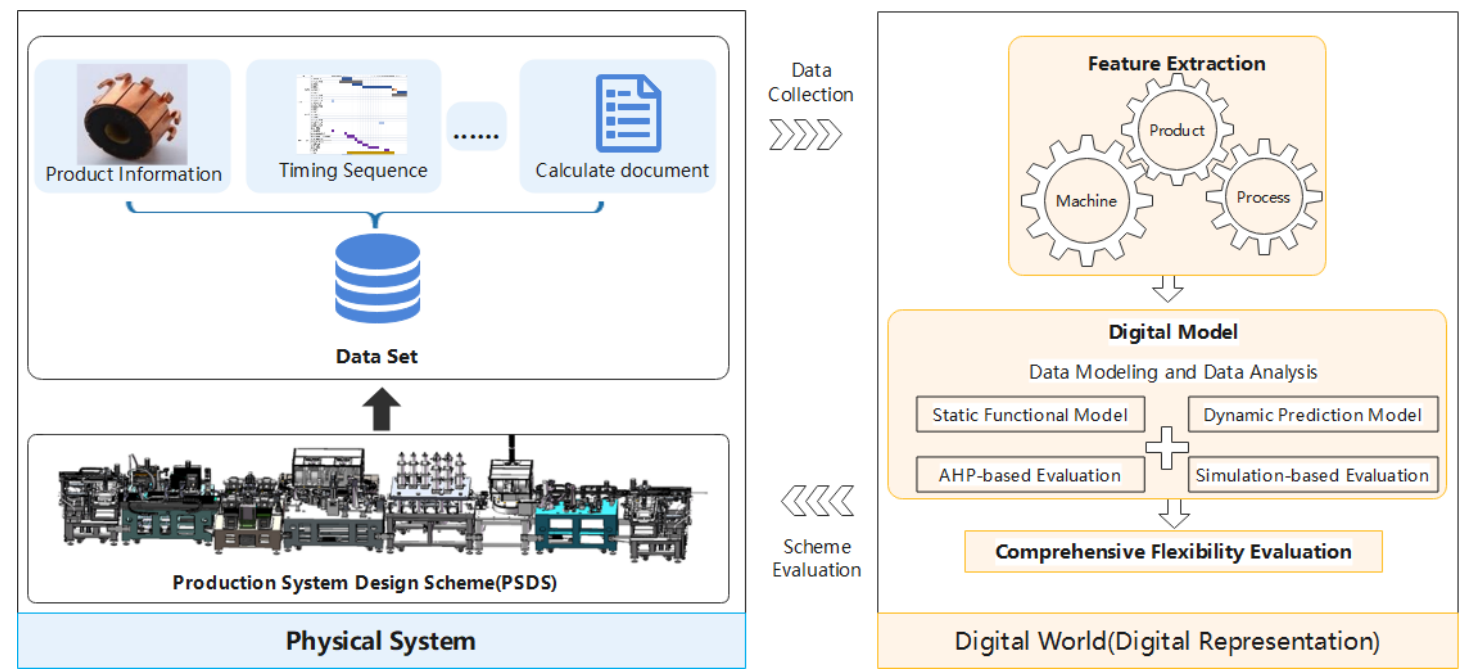

Figure 1. Production system flexibility research framework driven by digital twin.

The system flexibility research framework consists of three main subjects. The first subject is the physical system, which stores all design documents including the design scheme of the production system, and the design documents include the design requirements for new products production, design documents, calculation documents, design models, etc. These documents record the whole process of the production system from the initial requirement analysis to the generation of the demand-oriented design scheme. These are the original datasets to realize the flexible digital expression of the system.

The second subject is the digital world, which is the digital expression of system flexibility. Flexibility is the observable and research object defined in ISO/DIS 23247-1 standard. All aspects of it can be digital expressed with the support of digital twin technology, and can keep the same update iteration frequency with the physical object. Asma Ladj [22] adopted digital shadow to assist decision making in the machining process through data management and analysis. The constructed process-oriented digital shadow includes the data model and data analysis of the process data. The ISO/DIS 23247-3 standard proposes that the information contained in the observable object includes not only static but also dynamic information. For this reason, the digital model constructed in this paper adopts data models and data analysis methods in the process of digital expression oriented to system flexibility, and the system flexibility features concerned include static and dynamic dimensions. The data model is used to extract the characteristic parameters related to 
the system flexibility performance analysis in the original data set, and form a flexibility evaluation model based on the analysis of these characteristic parameters. Data analysis put forward an analysis method on the data model, and realized the quantitative evaluation of the flexibility evaluation model from dynamic and static dimensions to help analyse system flexibility.

The third subject is the information channel, which is to realize data interaction between the physical world and the digital world. On the one hand, it helps to realize the data extraction and collection related to the analysis of system flexibility characteristics from the physical world to the digital world, and on the other hand, it feeds back the analysis and evaluation results of system flexibility realized in the digital world to help the evaluation and development of the demand-oriented production system in the physical world. However, due to the limitation that it is only in the design phase, the virtual and real world cannot automatically realize data interaction and sharing, so the feature extraction, evaluation, and analysis results of the original data are carried out manually in the research framework of the system flexibility.

\section{System Flexibility Digital Representation}

The prerequisite for realizing system flexibility expression in all aspects is to realize a comprehensive analysis of the system characteristics, so that we can clearly understand the flexibility characteristics and then describe the system flexibility. Thus, in the process of digital expression of system flexibility, it is necessary to analyse the system flexibility first, clarify what characteristics can be used to express the flexibility of the production system in design phase, and what indicators are used to measure flexibility performance. Based on the comprehensive analysis of system flexibility, the system flexibility digital expression can be implemented according to the proposed architecture. Therefore, this section following content establishes a digital model describing system flexibility based on analysing system features.

\subsection{Flexibility Definition and Analysis \\ (1) Flexibility Definition}

Flexibility is a complex and multidimensional concept, which has different definitions and meanings in different application scenarios. In product innovation research, Adegoke Oke [5] emphasizes labour flexibility and mix flexibility. Labour flexibility exposes the workforce to a broad range of manufacturing tasks, and mix flexibility helps to quickly produce or develop different new product ideas to influence product innovation performance. Wei Zhong [23] defines flexibility as the balance management ability between energy storage and various heat sources (including renewable energy) in the heating demand in the study of the flexibility for the thermal system network. In the robot manufacturing system for SME, Chen Zheng [24] pays more attention to flexibility in customization and design. Flexibility in customization allows SMEs to customize the required manufacturing system by themselves. Flexibility in design [25], helps to develop new manufacturing systems easily by making appropriate changes from the existing ones. Tullio Tolio [26] considers the correct configuration or reconfiguration ability of the production system is the key technology in the flexibility transmission line design to meet the demand for new product characteristics change. Due to its multidimensional characteristics, researchers, especially the ones in the manufacturing area, identified many different but related classes of flexibility.

The flexibility studied in this paper is mainly considering the ability of the production system to meet the demand for new products' production. The needs of a production system for an enterprise cover many aspects, such as efficiency, cost, performance, etc. If the production system can obtain high production efficiency and production performance at low costs, it indicates that the design scheme has higher flexibility. However, there is no positive correlation between the cost, efficiency and performance of the system. If the production system wants to achieve high efficiency, they must give up the desire for low 
cost, and high production performance in most cases loses part of the production efficiency. It can be seen that the relationship between the various needs of the production system for the enterprise is more complicated. Therefore, we only consider part of the requirements that the enterprise needs when evaluating the flexibility for the production system in the design phase, which include the ability to meet the multiple varieties, small batches and quick switch of products' production demands, etc. This ability, on the one hand, is expressed as the ability of the production system to meet the needs of the company's products, processes, and machines, which can be obtained through the analysis of the structural attributes for PSDS, which is called the PSDS static characteristic performance, on the other hand, it is expressed as the ability of the production system to meet the changes in demand that may occur in the enterprise can be obtained by simulation and prediction for the PSDS, which is called the dynamic characteristic performance of PSDS.

(2) System flexibility characteristics analysis

In a review article, Jain summarized dozens of flexibility types and made corresponding definitions for different flexibility types. The summarized flexibility types for the manufacturing industry include machine flexibility, operation flexibility, path flexibility, volume flexibility, product flexibility and so on. In the existing studies, scholars often evaluate system flexibility performance by measuring a certain dimension flexibility to help system development. Baykasoğlu [27] takes machine flexibility as an evaluation index for production system flexibility, and considers machine operation efficiency, function conversion probability, function conversion efficiency and number of operable states as characteristic parameters to evaluate system flexibility. Oke [28] believes that labor flexibility enables labor to be exposed to a wide range of manufacturing tasks and can expand the skill and knowledge warehouse of labor. Therefore, hybrid flexibility and labor flexibility are taken as the characteristics of product innovation study. Real-time capability, quality of service, adaptive range and user interaction were considered by Vogel-Heuser [29] as evaluation indexes for system reconfigurable flexibility, and a digital evaluation model is constructed to quantify the index by focusing on the fault compensation capability of the system.

According to the definition for system flexibility, the production system flexibility in the scheme design phase is mainly manifested as the ability of the production system to meet the demand for producing new products. The system flexibility level is reflected by the system's inherent capability, which can be obtained by analyzing the system structural attributes. Therefore, the Unified Modelling Language (UML) is utilized to construct the system descriptive metamodel to describe system generic attribute information in the design phase by presenting the attribute information of different system components and the relationship between them. UML can standardize and visualize the whole process of entities, properties, relations, structures, states and dynamic changes of things $[30,31]$. Furthermore, UML provides mechanisms that enable new kinds of modelling elements to be defined and also enable relate the information to new modelling elements [32,33].

In the design phase, the most basic and important components include: product component, process component and machine component [34]. System descriptive metamodel describes system generic attribute information in the dimension of product, process and machine in Figure 2. In Figure 2, 1.. * indicates that there is at least one instance, 1 indicates that only one instance can be created. The product component covers the whole product information of the production system, and the basic building block is the product variety. A product component consists of one or more product varieties, and these product varieties belong to different product families. The process component reflects the process operation information, including the process operation capacity, process operation sequence, etc. The machine component covers all machine-able equipment types, machine processing capacity, machine layout, machine conversion capacity and other machine information owned by the production system. 


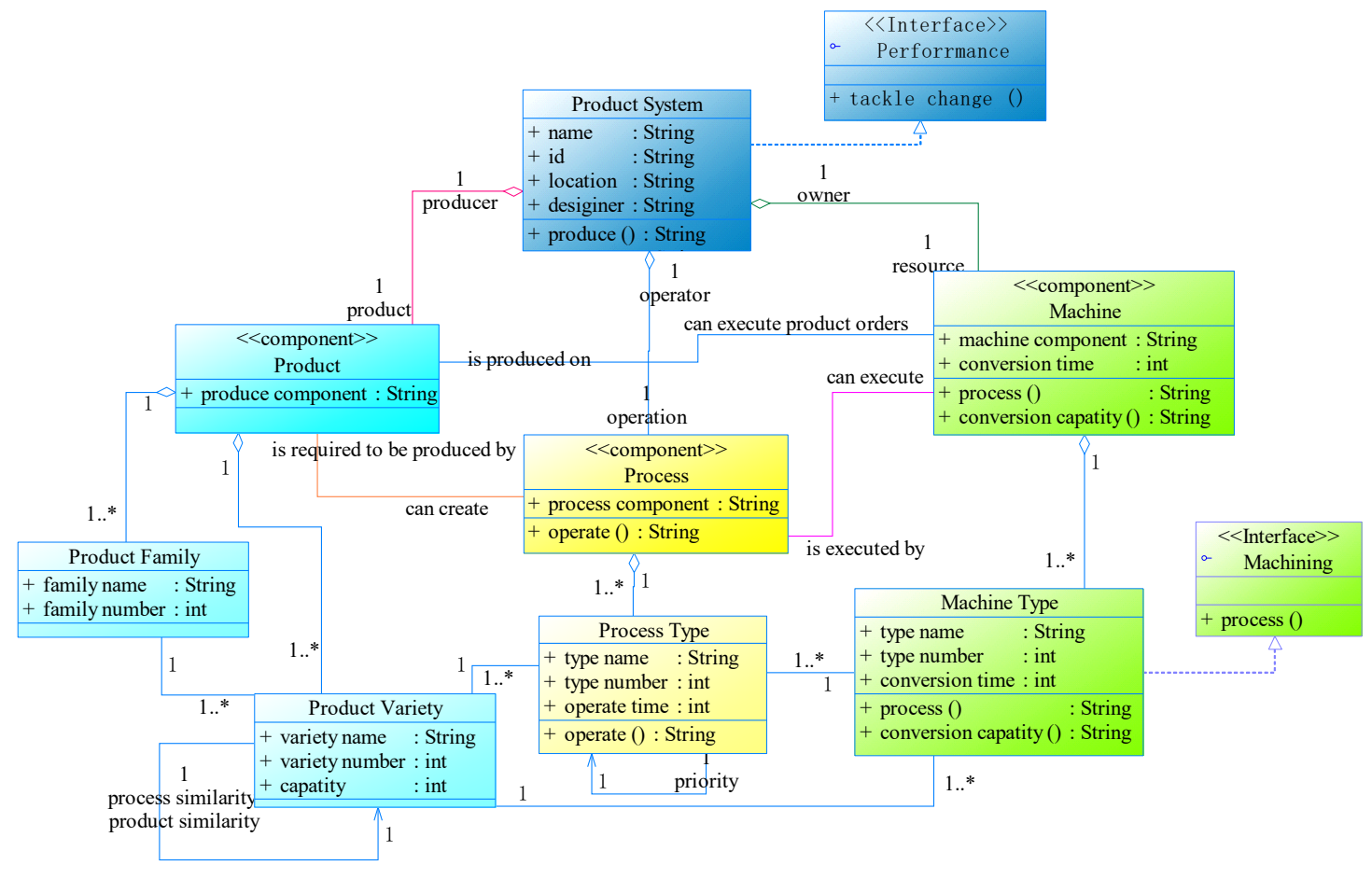

Figure 2. Production system structure descriptive metamodel.

Therefore, combining with the specific description of production system structural attributes, this paper puts forward the static characteristic evaluation metrics for production system flexibility from product, process, and machine dimension. The product dimension metrics include: number of product varieties, product similarity. The process dimension metrics consist of process changeability and process conversion capacity. Machine dimension metrics comprise machine versatility and machine extensibility. The definition of the metrics is described in Table 1, and the relationship between static evaluation metrics and system flexibility is also shown.

Table 1. Metrics definition and Relationship between metrics and system flexibility.

\begin{tabular}{lll}
\hline & Definition & $\begin{array}{l}\text { System } \\
\text { Flexibility }\end{array}$ \\
\hline Product varieties & $\begin{array}{l}\text { types of products produced by the system } \\
\text { similarity level of the products in structural } \\
\text { and process } \\
\text { product similarity }\end{array}$ & + \\
Process changeability & $\begin{array}{l}\text { efficiency of the production system to convert } \\
\text { between two different product varieties } \\
\text { Process conversion capacity }\end{array}$ & + \\
Machine versatility & $\begin{array}{l}\text { versatility level of the machine } \\
\text { extensibility ability of the production system } \\
\text { when facing the introduction of new }\end{array}$ & + \\
Machine extensibility & $\begin{array}{l}\text { products } \\
\text { '+: Positive correlation; '-': Negative correlation }\end{array}$ \\
\hline
\end{tabular}

According to the production system flexibility definition in the design phase and the system characteristic performance, only the product similarity metrics are inversely proportional to the system flexibility. Product similarity represents the structure between the types of products that the production system can produce. The higher the similarity between the products that the production system can produce, the lower the capacity of the production system to produce different kinds of products. Other characteristic metrics are proportional to the system flexibility. 
The number of product types refers to the number of product types that can be produced by the production system. The higher the quantity of product types produced, the stronger the ability of the production system design scheme to meet the needs of multivariety product production. Product similarity refers to the similarity level of the products in structural and process. The lower the similarity level between the products that can be produced by the production system, the higher capacity for the production system to produce different types of products. Process changeability refers to the changeability index for product process path. The more process paths a product can choose in the production process, the higher the probability that the system can continue the production when a certain equipment fails, which means the greater production system flexibility. Process conversion capacity refers to the efficiency of the production system to convert between two different product varieties, which is proportional to the system flexibility. Machine versatility refers to the versatility level of the machine. Machine in mechanical processing can be divided into dedicated machines (such as boring machine saw machine) and general machines (such as CNC, Turning and milling compound, etc.). A dedicated machine is customized for a specific processing process and has the ability to operate only one process, and general machine is capable of two or more processes. If the production machine in the system has a higher degree of versatility, it shows that the system's inherent ability is higher as well as its flexibility. Machine extensibility refers to the extensibility ability of the production system when facing the introduction of new products. The stronger the expansion capability, the greater flexibility.

Production systems in the design phase should not only have the ability to meet the needs of products, processes, and machines for new product production, but also have the ability to respond to environmental changes. In Figure 2, the production system has the ability to respond to changes in demand. For this reason, environmental adaptability is defined as a flexibility dynamic evaluation metric for PSDS. The environmental changes that the production system may encounter include internal and external environmental changes. Changes in the internal environment include device failure, tool wear, transmission congestion, etc. Changes in the external environment include production orders insertion emergency, raw materials insufficient supply, etc. Considering that this paper is a study on the design phase of the production system, we believe that the most likely or predictable environmental change is device failure within the production system. Therefore, the flexibility dynamic evaluation for PSDS mainly studies the response ability of the system to the possible device failure rate. The stronger the capability is, the higher the flexibility level of the system will be.

\subsection{Flexibility Feature Extraction \\ Physical System for Data Collection}

The physical system for flexibility evaluation composes several design schemes, which are designed according to the requirements of new products production. In accomplish the PSDS, the detailed design requirements, design documents, design models and other design data constitute a complete physical system, and the information and data used for the flexibility evaluation can be extracted from the physical system.

Flexibility metrics for system static and dynamic evaluation are proposed. Static evaluation metrics are proposed in consideration of the system's structural attributes, and dynamic evaluation metrics focus more on the ability of the system to respond to environmental changes. According to the proposed static evaluation metrics and combined with the production system structure description metamodel, the basic static feature parameters describing the flexibility of the PSDS are defined, which include product variety $v_{i}$, machine type $m_{k}$, process type $p_{j}$, product family $v t y_{f}$, production throughput $c_{i}$, and process adjustment time $t_{h}$. The static characteristic parameters describe the various dimensions characteristics of the flexibility for the PSDS. The physical system consists of lots of design data, such as product design requirements after multiple iterations, customized machine, process design for different products, etc., which constitute the source data for 
the quantitative extraction of static feature parameters. Environmental adaptability is defined as the dynamic evaluation metric, and the device failure cause for process losing is assumed to be the most occurred internal environment change, which leads to system breakdown. The flexibility dynamic evaluation of PSDS is defined as the system production capacity in the face of possible dynamic changes of device failures. The device failure rate $\mathrm{mal}_{r}$ is taken as the characteristic parameter for the flexibility dynamic evaluation, which is a random variable. The data collected for the flexibility dynamic evaluation includes the equipment layout extracted from the design model, the timing data designed by the production volume, and the production process determined by the production demand.

\subsection{Data Modelling and Data Analysis}

The digital twin flexibility evaluation (DTFE) is mainly responsible for processing, modelling, and analysis and decision making of the collected data. The collected data comes from the constructed physical system, and the data information to be collected is positioned to the requirements of the system characteristic parameters. The model for DTFE consists of the digital twin static function model for system static characteristic parameters and the digital twin dynamic prediction model for system dynamic characteristic parameters. The static function model for system flexibility needs to define a series of parameters:

$v v_{i l}$ : judgment value of whether product variety $v_{i}$ and product variety $v_{l}$ are the same family. If $v_{i}$ is of the same family as $v_{l}$ or $i=l$, then take $v v_{i l}$ as 1 , and 0 otherwise;

$v p_{i j}$ : judgment value of whether process type $p_{j}$ is required for manufacturing product $v_{i}$. It assumes value 1 if process $p_{j}$ is required for the production of product $v_{i}$, and 0 otherwise;

$p m_{j k}$ : judgment value of whether the process type $p_{j}$ is processed by the machine type $m_{k}$. It assumes value 1 if the process type $p_{j}$ is processed by the machine type $m_{k}$, and 0 otherwise;

$p p_{i l}$ : the quantity of the same process type that product type $v_{i}$ and product type $v_{l}$ have, and $p p_{i l} \leq p p_{i i}$.

$p t_{s j}$ : the judgment value of whether the sequence between process type $p_{s}$ and process type $p_{j}$ is changeable. When $s$ does not equal to $j$, and the sequence of process $p_{s}$ and $p_{j}$ can be changed, then taken $p t_{s j}$ as 1 , and 0 otherwise. When $s$ is equal to $j$, the sequence changeability between two identical processes represented by $p t_{s, j}$ is meaningless in the actual production process, so take $p t_{s, j}$ as 0 ;

$m m_{k o}$ : judgment value of whether the machine type $m_{k}$ and the machine type $m_{o}$ are pluggable. When $k$ is not equal to $o$, it assumes value 1 if the machine type $m_{0}$ is arranged in parallel with the machine type $m_{k}$, take $m m_{k o}$ to be 1 and 0 otherwise;

$v c_{i}$ : output of $v_{i}$ produced by the production system per hour, and the $V Q$ matrix is a diagonal matrix;

$t r_{i I}$ : adjustment time required by the production system to make production transformation between products $v_{i}$ and product $v_{I}$;

$S i_{v_{i}, v_{h}}$ : similarity value between product variety $v_{i}$ and product variety $v_{h}$, the greater the value, the higher the similarity. if $i=l$, then $S i_{v_{i}, v_{h}}=1$, and if $i \neq l$, then $S i_{v_{i}, v_{h}} \in[0,1]$.

\subsubsection{Static Functional Model (SFM)}

The static function model (SFM) mainly describes and analyses the system static characteristic parameters, so as to evaluate the ability of the PS to meet the enterprise's requirements. The model focuses on the system feature analysis network (SFAN) described in Figure 3, which integrates and analyzes the static characteristic parameters according to the different interaction relationships of the product, process, and machine dimensions, and combines the proposed flexibility static evaluation metrics to form a multidimensional digital twin flexibility evaluation model. The multidimensional digital twin flexibility evaluation model covers a product-dimension flexibility evaluation model, process-dimension flexibility evaluation model, and machine-dimension flexibility evaluation model. 


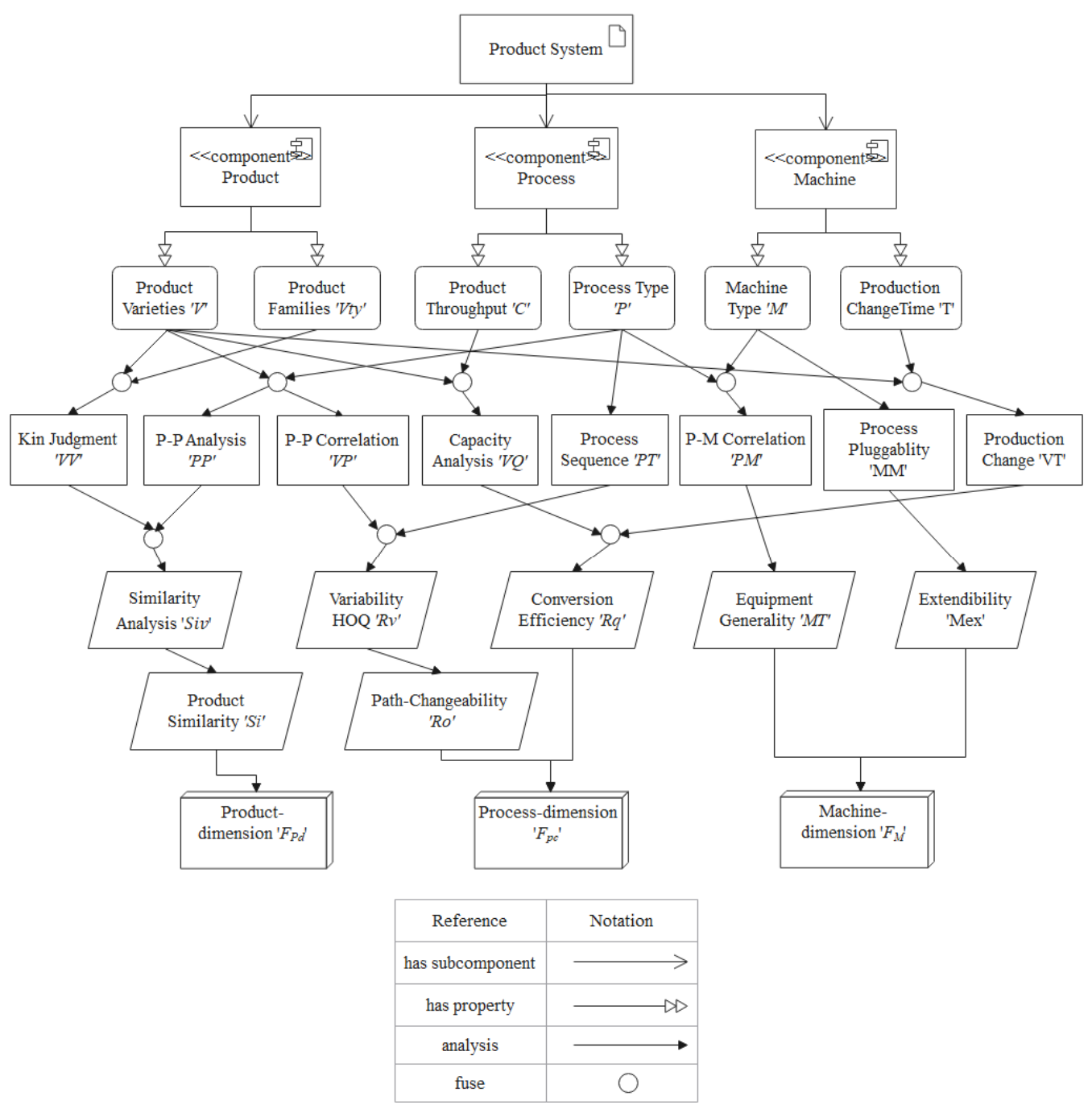

Figure 3. System feature analysis network.

With analyzing the relationships and properties of the components described in the descriptive metamodel, the characteristic matrices are defined in Equations (1)-(8), they are: product family judgment matrix $V V$; product process correlation matrix $V P$; product machine correlation matrix $P M$; product process analysis matrix $P P$; process sequence changeability matrix $P T$; process insertability matrix $M M$; product conversion time analysis matrix $V Q$; conversion time analysis matrix $V T$.

$$
\begin{gathered}
V V=\left(v v_{i l}\right)_{I \times I} \quad \forall i, l \in[1, I] \\
V P=\left(v p_{i j}\right)_{I \times J} \quad \forall j \in[1, J] \\
P M=\left(p m_{j k}\right)_{J \times K} \quad \forall k \in[1, K] \\
P P=\left(p p_{i l} / p p_{i i}\right)_{I \times I} \\
P T=\left(p t_{s j}\right)_{J \times J} \quad \forall s, j \in[1, J] \\
M M=\left(m m_{k o}\right)_{K \times K} \quad \forall k, o \in[1, K] \\
V Q=\left(v c_{i}\right)_{I \times I} \\
V T=V T_{I \times I}=\left(t r_{i l}\right)_{I \times I}
\end{gathered}
$$


Product dimension flexibility aims at analysing the similarity and the number of product varieties that a PS can produce. First, the structural and process similarity analysis matrix $S i_{v}$ of the product is obtained by analyzing the product homology relationship and processing technology of all products.

$$
\begin{gathered}
S i_{v}=0.5(P P+V V)=\left(S i_{v_{i}, v_{h}}\right)_{n \times n} \\
S i_{v_{i}, v_{h}}=0.5\left(p p_{i l} / p p_{i i}+v v_{i l}\right)
\end{gathered}
$$

Combined with the quantity of product varieties that can be produced by the production system and the product similarity analysis matrix, the product similarity value $S i$ is obtained, and $S i$ can be given by

$$
S i=\left(\sum_{h=1}^{n} \sum_{i=1}^{n} S i_{v_{i}, v_{h}}-Q_{V}\right) / 2 C_{Q_{V}}^{2} S i \in[0,1]
$$

The flexible evaluation of product dimension can be analysed from two aspects. For one thing, the more product families the production system can produce, the more flexibility the production system will be. For another, the lower the similarity between product varieties, the higher the flexibility of the production system. Therefore, the product dimension flexibility evaluation model $F_{P d}$ can be represented as:

$$
F_{P d}=Q_{V t y} / 2 Q_{V}+(1-S i) / 2 \quad F_{P d} \in[0,1]
$$

Process dimension flexibility is mainly determined by the adjustment degree of the processing technology for the existing production system structure, including the changing ability $R_{o}$ of the processing path for each product and the production efficiency $R_{q}$ of the production system to transform production between different product varieties. The calculation of $R_{o}$ for the changeability of the product processing path includes the house of quality model of the process path variability $(R v)$, which is represented in Figure 4 .

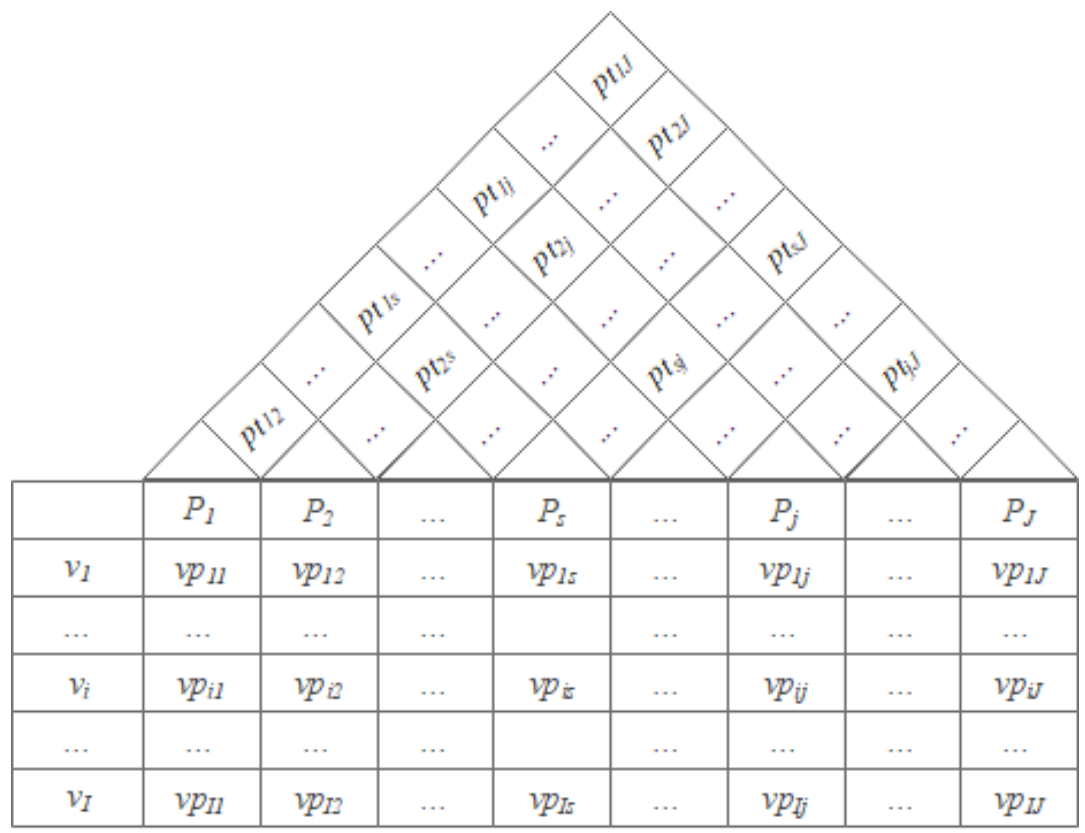

Figure 4. The house of the process path variability.

Analysing the rank relationship of the process path variability quality house model, the quantity of alternative paths for product variety $r_{v_{i}}$ is obtained. Thus the process path 
changeability of the production system $R_{o}$ is obtained by calculating the number of process paths of all product varieties.

$$
\begin{gathered}
r_{v_{i}}=1+\sum_{s=1}^{J} \sum_{j=1}^{J} p t_{s j} \times v p_{i j} \times v p_{i s} \quad j \neq s \\
R_{o}=\left(\sum_{i=1}^{I} r_{v_{i}}-Q_{V}\right) / \sum_{i=1}^{I} r_{v_{i}} R_{o} \in[0,1]
\end{gathered}
$$

The calculation of $R_{q}$ is combined with product capacity analysis matrix $V Q$ and product conversion time analysis matrix $V T$, the production loss of production system conversion $R_{q l o \_}$at any time $t$ is used to evaluate the production conversion efficiency for the production system in the limit of $8 \mathrm{~h}$ working time per day. And

$$
\begin{aligned}
& R_{q l o_{i l}}=\left[t \times v c_{i}+v c_{l}\left(8-t-t r_{i l}\right)\right] /\left[t \times v c_{i}+v c_{l} \times(8-t)\right]=1-v c_{l} \times t r_{i l} /\left[t \times v c_{i}+v c_{l} \times(8-t)\right] \\
& \text { where } R_{q l o \_i l} \in[0,1] . \text { Thus, } \\
& R_{q}=\sum_{i=1}^{I} \sum_{l=1}^{I} R_{q l o \_i l} / A_{I}^{2}
\end{aligned}
$$

The process dimension flexible evaluation model $F_{P_{c}}$ is obtained by the combination of $R_{o}$ and $R_{q}$, which can be represented as:

$$
F_{P c}=0.5\left(R_{o}+R_{q}\right)
$$

The machine dimension flexibility mainly describes the machine versatility and machine extensibility of the production system. The versatility of a single machine is defined, then the machine versatility of the production system is defined too.

$$
\begin{aligned}
& M T_{k}=\left\{\begin{array}{cl}
0 & , \text { when } Q_{P}=1 \\
\left(\sum_{j=1}^{J} p m_{j k}-1\right) /\left(Q_{P}-1\right) & \in[0,1], \text { when } Q_{P}>1
\end{array}\right. \\
& M T=\sum_{k=1}^{K} \mathrm{MT}_{k} / Q_{M} \quad M T \in[0,1]
\end{aligned}
$$

Machine extensibility represented as:

$$
\operatorname{Mex}=\sum_{k=1}^{K} \sum_{o=1}^{K} m m_{k, o} / 2 C_{Q_{M}}^{2} \quad \text { Mex } \in[0,1]
$$

Finally, the machine dimension flexible evaluation model can be defined as:

$$
F_{R M}=0.5(M e x+M T) \quad F_{R M} \in[0,1]
$$

\subsubsection{Dynamic Prediction Model (DPM)}

Dynamic prediction model (DPM) is used to predict processing capacity of the production system (PS) in the face of device failure, and the model structure is shown in Figure 5. Each device in the PS has the possibility of failure during production. For processing devices, possible faults include tool wear, tool damage, machining accuracy deviation, etc., while for the material transfer devices, the possible failures include material loss, material pick up and put wrong, etc. Therefore, the Digital Twin Dynamic Prediction Model (DTDPM) in Figure 5 takes the random device failure rate as the input parameter of the model, and takes the system dynamic performance description model as the main body, considers the throughput of each schemes under the present device failure rate as the output, which is described by Formula (22).

$$
\text { DTDPM }=\{\text { Designed malfunction, System dynamic property, Predictive performance }\}
$$




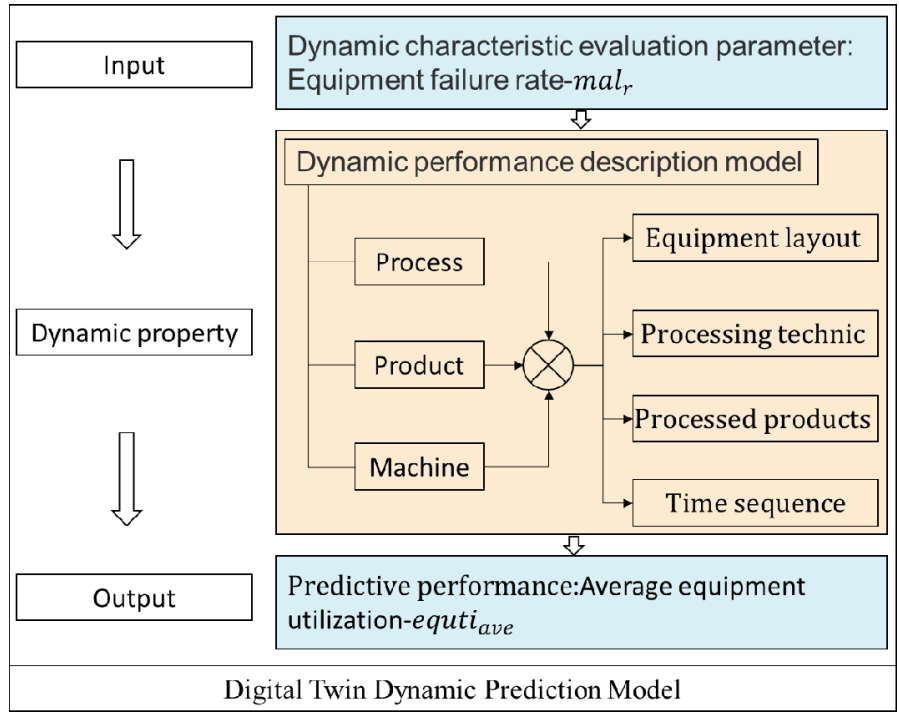

Figure 5. Structure diagram of dynamic prediction model.

System dynamic property is a description model of the system dynamic performance, which is a data set collected from process, product and machine dimensionality cover the information of equipment layout, processing technology, processing product category, processing time sequence and other information reflected in the design scheme of the production system as in Formula (23). In the production system structure description metamodel, the basic structure of this information is described in detail. In the face of a specific production system design scheme, this information is all the instance information in the description metamodel.

\subsubsection{AHP-Based System Flexibility Static Evaluation Index}

The digital twin flexibility evaluation of production system design scheme (PSDS) is designed to obtain the flexibility evaluation quantitative index. The flexibility static evaluation index can be obtained through expert scoring and AHP method combined with the analysis of digital twin static functional model. The production system scheme designed for the demand of the enterprise has different characteristic attributes, and the staffs have different production tasks in the production enterprise have different subjective requirements for system flexibility. The expert score and AHP method are combined to obtain the enterprise's weight for the flexibility of product, process, and machine in the system static function model.

To obtain flexibility weight, the judgment matrix needs to be constructed first. Judgment matrix is mainly considering production enterprise's subjective demand for flexibility in the design phase. Invite enterprise staffs (e.g., process engineering, production manager, etc.) to grade the relative importance of three-dimensional flexibility with their own understanding of the enterprise acquirements on the basis of the score standards in Table 2. The mean value of the scoring results is obtained to obtain the positive reciprocal matrix A.

$$
\mathrm{A}=\left(a_{x y}\right)_{3 * 3} \quad \forall x, y \in[1,3]
$$

where, $a_{x y}$ represents the importance of a dimension compared with another dimension in the factors affecting the flexibility of the production system. For the judgment matrix, combined with the normalization processing method, the flexibility weight of each dimension is expressed.

$$
W=\left(W_{1}, W_{2}, W_{3}\right)^{T}=\sum_{y=1}^{3} \overline{W_{x y}} / \sum_{x=1}^{3} \sum_{y=1}^{3} \overline{W_{x y}}
$$




$$
\bar{W}_{x y}=a_{x y} / \sum_{x=1}^{3} a_{x y}
$$

where, $W_{1}, W_{2}$ and $W_{3}$ are the weights of the influence factors for product dimension flexibility, process dimension flexibility, and machine dimension flexibility respectively. The flexibility static evaluation index $F L$ of the production line is obtained by using linear weighting:

$$
F L=W_{1}, W_{2}, W_{3} \times\left(F_{P d}, F_{P c}, F_{R M}\right)^{T}=W_{1} \times F_{P d}+W_{2} \times F_{P c}+W_{3} \times F_{R M}
$$

Table 2. Grade criterion.

\begin{tabular}{ll}
\hline Importance Degree & Grade Criterion \\
\hline Equal important & 1 \\
General important & 3 \\
More important & 5 \\
\hline
\end{tabular}

\subsubsection{Simulation-Based System Flexibility Dynamic Evaluation Index}

Based on the simulation platform Plant Simulation, the proposed digital twin dynamic prediction model is constructed to obtain the flexibility dynamic evaluation index. The simulation is carried out under the device failure rate $\mathrm{mal}_{r}$, and the system production capacity $Q_{\text {output_r } r}$ can be obtained at a certain time. The system flexibility dynamic evaluation index is the production stability of the system in the face of possible failures, which is shown as the fluctuation of the production volume in the face of device failures. The larger the fluctuation is, the worse the ability of the system to deal with failures will be.

$$
Q_{\text {output_r }}^{\prime}=\left(Q_{\text {output_r }}-\operatorname{Min}\right) /(\operatorname{Max}-\operatorname{Min})
$$

Equation (28) is the normalization process of production volume at different failure rates obtained by different schemes. Min is the minimum value of production in each group of simulation results, and max is the maximum value of production in each group of simulation results.

$$
F L_{\text {dynamic }}=\sum_{1}^{K} D\left(Q_{\text {output_r }}^{\prime}\right) / K
$$

\subsubsection{Comprehensive Flexibility Evaluation Index}

The comprehensive flexibility evaluation index of the production system shows the ability of the design scheme of the production system to quickly switch the production types of multiple varieties and small batches of products in the demands of the enterprise. It is the weighted integration of the static flexibility evaluation index and the dynamic flexibility evaluation index of the production system, and its calculation expression is:

$$
F L=F L_{\text {static }} / F L_{\text {dynamic }}
$$

\section{Case Study}

To evaluate the feasibility and effectiveness of the proposed method, this section shows the application case in related enterprises. The motor commutator is an important part of DC motor and AC commutator motor, which plays a reversing role when the motor rotates. $\mathrm{KZ}$ is a motor commutator manufacturer, and its products are diversified, which are in great demand at home and abroad. The existing production pattern of the enterprise has low automation degrees, high labour cost and low device utilization rate. However, with higher demands put on the manufacturing capabilities of enterprises, the traditional production mode of the enterprise cannot meet the demand of the short cycle, multivariety and small batch production. The enterprise is in urgent need of changing the existing production mode, reducing labour cost and improving the rapid response ability of the enterprise to new products. To this end, the target enterprise (Hereinafter referred to as $\mathrm{HC}$ ) 
proposes two production system design schemes, A and B, according to the requirements of the motor commutator manufacturer. The machining process of the motor commutator is: inner bore turning, outer circle turning, slot milling, hook, and the required product quality inspection includes: inner bore size accuracy, outer circle size accuracy, slot size accuracy, and processing surface quality inspection. A brief introduction to scheme A and $\mathrm{B}$ is as follows.

In scheme A, the loading and unloading of workpieces is realized manually. After the loading is completed, the transport parts transfer the workpiece to the process machine. After the workpiece has completed all the processing and inspection processes, the unloading machine puts the workpieces on the tray one by one, and manually pushes the loading trolley to the packing place.

In scheme B, the workpieces are placed by the workers in the warehouse. Then the AGV will automatically pick up the workpiece from the warehouse and transport it to the corresponding machine when receiving the control command. After the processing is finished, the AGV takes the next step according to the control command. AGV may be required to send boxes containing semifinished parts to the next machine or to return boxes containing finished parts to the warehouse.

\subsection{Flexibility Evaluation}

(1) System Flexibility Characteristics

To realize the digital expression of multidimensional system flexibility, the first step is to extract the data of flexibility feature in the system design scheme. According to the analysis of the two design schemes, the basic static characteristic parameters used to describe the flexibility in production system design schemes are extracted, part of parameters are shown in Table 3. Scheme A can produce four types of products, which belong to the same product family. Scheme A has 10 kinds of process capacity, and the number of device types is 9 . Among them, the number of types of machine with processing capacity is seven (feeding machine and discharging machine are not counted). Scheme B can produce five types of products, which belong to two product families. Scheme B also has 10 kinds of process capacity, and the number of device types is 9 . Among them, the number of types of machine with processing capacity is eight (AGV is not counted). For the following flexibility analysis, a random failure rate of $0 \sim 20 \%$ was set for each processing machine, with a failure rate increase of 0.5 . The hourly output of each product in scheme A and $\mathrm{B}$ is 1028 pieces, and different production layouts and timing are given in the design documents of the two schemes.

Table 3. Scheme A and B: basic flexibility static characteristic parameters.

\begin{tabular}{|c|c|c|c|c|}
\hline & $Q_{V}$ & $Q_{P}$ & $Q_{M}$ & $Q_{V t y}$ \\
\hline Plan A & 4 & 10 & 9 & 1 \\
\hline Plan B & 5 & 10 & 9 & 2 \\
\hline
\end{tabular}

\section{(2) Data modelling and data analysis}

The characteristic parameters extracted from the production system design documents are converted according to the matrix (1)-(8) can obtain interaction relation matrix between the scheme A and B. Then, according to the requirements of flexibility static evaluation model, quantitative indexes of evaluation models of product dimension, process dimension and equipment dimension can be obtained, as shown in Table 4. According to the requirements of Equations (22) and (23), the dynamic prediction of system flexibility can be realized based on the equipment layout, processing technology, product types, processing time sequence, and other information extracted from the design document of the production system scheme. 
Table 4. Flexibility static evaluation index.

\begin{tabular}{ccccccccc}
\hline & Product-Dimension & \multicolumn{3}{c}{ Process-Dimension } & \multicolumn{3}{c}{ Machine-Dimension } \\
\hline & $\boldsymbol{S} \boldsymbol{i}$ & $\boldsymbol{F}_{\boldsymbol{P d}}$ & $\boldsymbol{R}_{\boldsymbol{o}}$ & $\boldsymbol{R}_{\boldsymbol{q}}$ & $\boldsymbol{F}_{\boldsymbol{P c}}$ & $\boldsymbol{M T}$ & $\boldsymbol{M e x}$ & $\boldsymbol{F}_{\boldsymbol{M}}$ \\
\hline $\mathrm{A}$ & 1 & 0.125 & 0 & 0.875 & 0.4375 & 0.0123 & 0 & 0.062 \\
$\mathrm{~B}$ & 0.79 & 0.33 & 0 & 0.875 & 0.4375 & 0.0123 & 1 & 0.062 \\
\hline
\end{tabular}

After feature extraction and modelling of the production system design scheme, data analysis can be carried out to evaluate the static flexibility of the system based on AHP and the dynamic flexibility of the system based on Plant Simulation platform. The dynamic flexibility evaluation model of the system design schemes are shown in Figure 6.

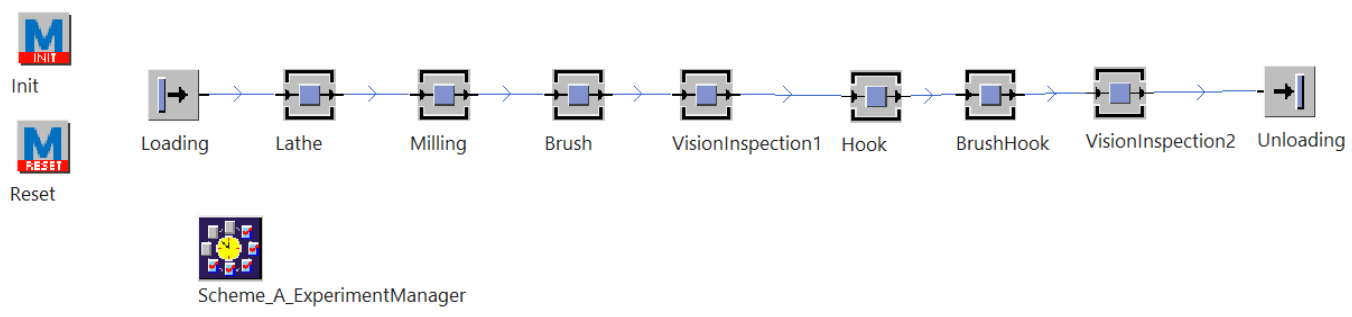

(a)

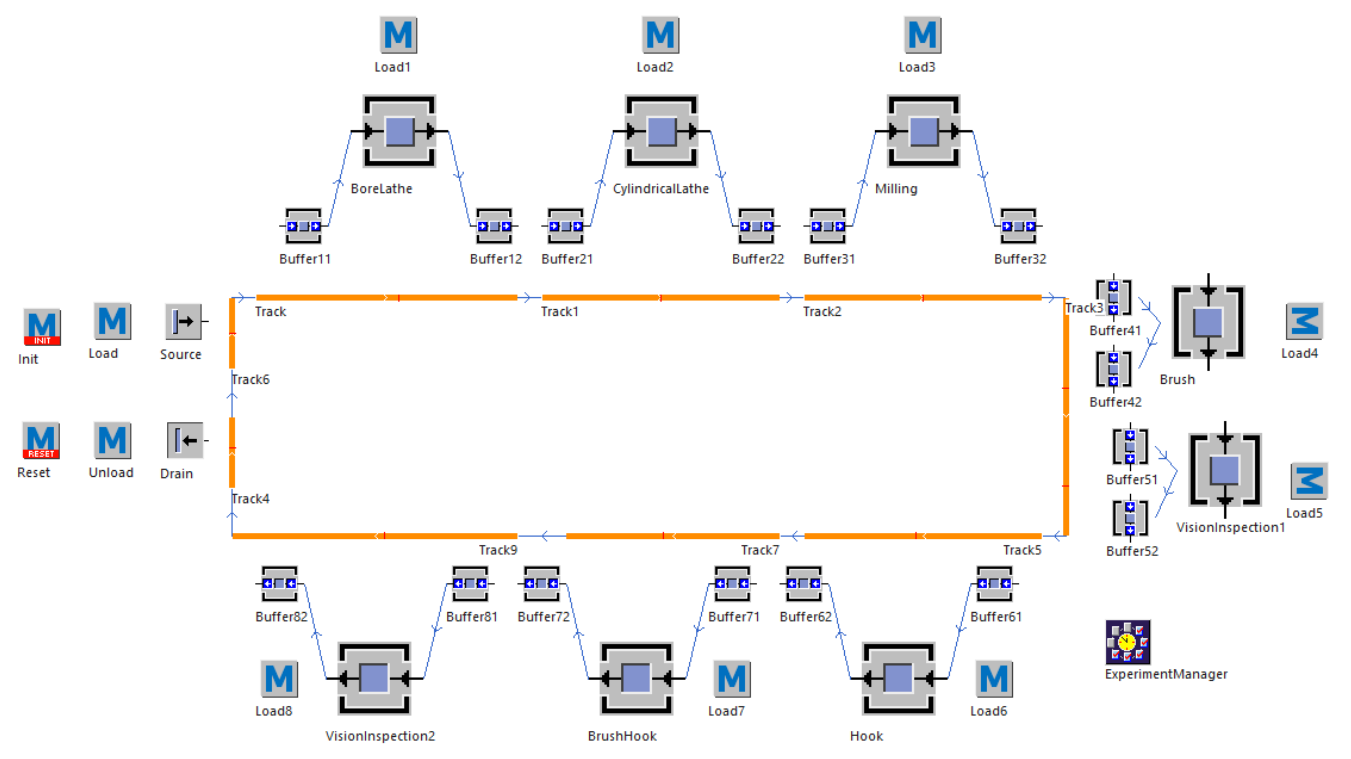

(b)

Figure 6. Dynamic prediction model of production system design scheme. (a) Dynamic prediction model for A. (b) Dynamic prediction model for B.

The flexibility static evaluation of the PSDS combines the subjective demand of the enterprise for flexibility production. Therefore, a questionnaire survey was conducted on 15 production personnel (including a production manager, process analyst, and product quality inspector) in the demand enterprise to obtain their evaluation on the importance of different dimensions of flexibility in system design. The evaluation results are summarized and analyzed according to Equations (24)-(26), which helps to obtain the flexibility weights of different dimension. The weights are $0.26102,0.21505$, and 0.52393 , respectively. Additionally, the static flexibility evaluation index in Table 5 can be obtained with the multi-dimensional flexibility evaluation index of the PSDS. 
Table 5. Flexibility result of commutator PSDS.

\begin{tabular}{lll}
\hline & A & B \\
\hline Static flexibility evaluation index & 0.12995 & 0.44542 \\
Dynamic flexibility evaluation index & 0.08596 & 0.071853 \\
Comprehensive flexibility evaluation index & 1.51175 & 6.19905 \\
\hline
\end{tabular}

In the flexibility dynamic evaluation of the PSDS, Scheme A has seven processing machines and Scheme $B$ has eight processing machines. The failure rate of each processing equipment is set as $0 \sim 20 \%$, and the failure rate increment is set as 0.5 . Therefore, 41 groups of experiments are carried out for each processing equipment, and each group is observed five times to obtain the simulation experiment of the production system production volume, and then the dynamic prediction results of two design schemes are obtained. Finally, the production capacity of each processing equipment at different failure rates is processed according to Equations (28)-(30), which obtains the comprehensive flexibility evaluation index of the production system design scheme in Table 5.

\subsection{Results Discussion}

Table 5 shows the quantitative evaluation results of the proposed evaluation method on the flexibility of the motor commutator production system design scheme. Flexibility in the case mainly considering the ability of the production system to meet the demand for new products' production. The comprehensive flexibility evaluation index in Table 5 represents the ability of different design schemes to meet the production requirements of new products. It can be seen that the comprehensive flexibility evaluation index of Scheme B is 6.19905, which is larger than 1.51175 in Scheme A. It means that Scheme B is more capable of meeting the needs of enterprises than Scheme A. The acquisition of the comprehensive flexibility evaluation index of the design scheme mainly considers two factors. One is the ability of the PS to meet the enterprise's requirements reflected by the system structure. The other one is the capacity of the production system (PS) to deal with equipment failure, obtained by simulation and prediction. The evaluation results show that the static flexibility evaluation index of Scheme A is 0.12995 and the dynamic flexibility evaluation index is 0.08596 , while the static flexibility evaluation index of Scheme B is 0.44542 and the dynamic flexibility evaluation index is 0.071853 . Numerical comparison indicates that the two design schemes have basically the same processing capacity in the face of possible equipment failure, but are quite different in meeting the production requirements of enterprises. The capacity of scheme $\mathrm{A}$ to meet the production requirements of enterprises is much smaller than that of Scheme B.

A holistic analysis of the case can be found, the two design schemes presented in this study have obvious contrasting characteristics, which also brings better verification effect to the application of the proposed digital twin flexibility evaluation framework. Comparing the values of the two schemes' characteristic parameters, Scheme B can produce more product varieties than $\mathrm{A}$, and Scheme $\mathrm{B}$ has the larger number of product families than that of Scheme A, while the quantity of process types and machine types are equal in Scheme $\mathrm{A}$ and Scheme B. Furthermore, Scheme A is a rigid production line, while Scheme B is constructed by independent production platforms which are linked by AGV. Therefore, it can preliminarily judge that Scheme B is more flexible than Scheme A, and the proposed flexibility evaluation method can better verify the predicted results.

According to the application results of the case, it can be found, based on the digital twin flexibility evaluation framework, the flexibility evaluation of the motor commutator PSDS is carried out quickly. The comprehensive flexibility evaluation indexes of the two design schemes not only consider the ability of the system itself to meet the production requirements of the enterprise, but also predicts the performance of the system in the face of possible failures. More importantly, the final evaluation results also integrate the enterprise's subjective demand for flexibility in the production process. 


\section{Conclusions}

To meet the needs of diversified markets in an ever-changing and turbulent market environment, a novel digital-twin-driven approach for rapid flexibility evaluation of production systems is proposed, which contribute to the production system design scheme (PSDS). The comprehensive flexibility evaluation takes into account both static and dynamic evaluation metrics; the static metrics are used to evaluate the system flexibility to the production demand, and the dynamic metric is used to predict the fault handling ability in the system design phase.

After that, a suitable digital-twin-driven evaluation framework is proposed. In the physical world, a UML-based system metamodel helps to analyse the flexibility in the system design stage which defines the features need to be extracted. Data modelling and data analysis are the focus of the digital world. The static function model and dynamic prediction model realized the flexible evaluation model construction of the original data set, while the data analysis realized quantitative evaluation of the flexibility from the passive and static dimensions with the help of AHP and Plant Simulation. The evaluation method proposed in this paper can not only quickly and accurately build the model, predict and evaluate the system flexibility, and help screen the optimal design scheme, but also can be used as a guideline for the improvement and optimization of the plan in the digital system design process.

Although the model is mainly applied to the design phase of the production system in this paper, if the production system production commissioning is completed, the model can then be applied to the operation phase of the production system. By establishing an automatic information interaction channel with the physical system, the model can obtain the system operation information in real time and form an online information processing mechanism to realize the flexibility digital twin construction of the system.

The method studied in this paper also has some limitations. The flexibility evaluated in this paper only considers the ability to meet part of the requirements for enterprise. However, in the design phase of the production system, the efficiency, cost and personnel of the production system are also important factors for enterprises to consider in the production of new products. If these factors are also taken into consideration in the system flexibility analysis, the production system flexibility evaluation will be more comprehensive, which is also the research direction for the future study of a more perfect production system comprehensive flexibility evaluation method.

Author Contributions: S.H. and B.L. contributed to the funding acquisition, K.L. and C.H. contributed to the background investigation and resources acquisition, L.Z. contributed to the methodological research and wrote the first draft of the manuscript, and X.M. responsible for the final review and editing. All authors have read and agreed to the published version of the manuscript.

Funding: This research is financially supported in part by the National Key R\&D Program of China under Grant [NO.2018YFB1700500]; and in part by the Key R\&D Program of Hubei China under Grant [NO.2020BAB106].

Institutional Review Board Statement: Not applicable.

Informed Consent Statement: Not applicable.

Data Availability Statement: Not applicable.

Conflicts of Interest: No potential conflict of interest was reported by the authors. 


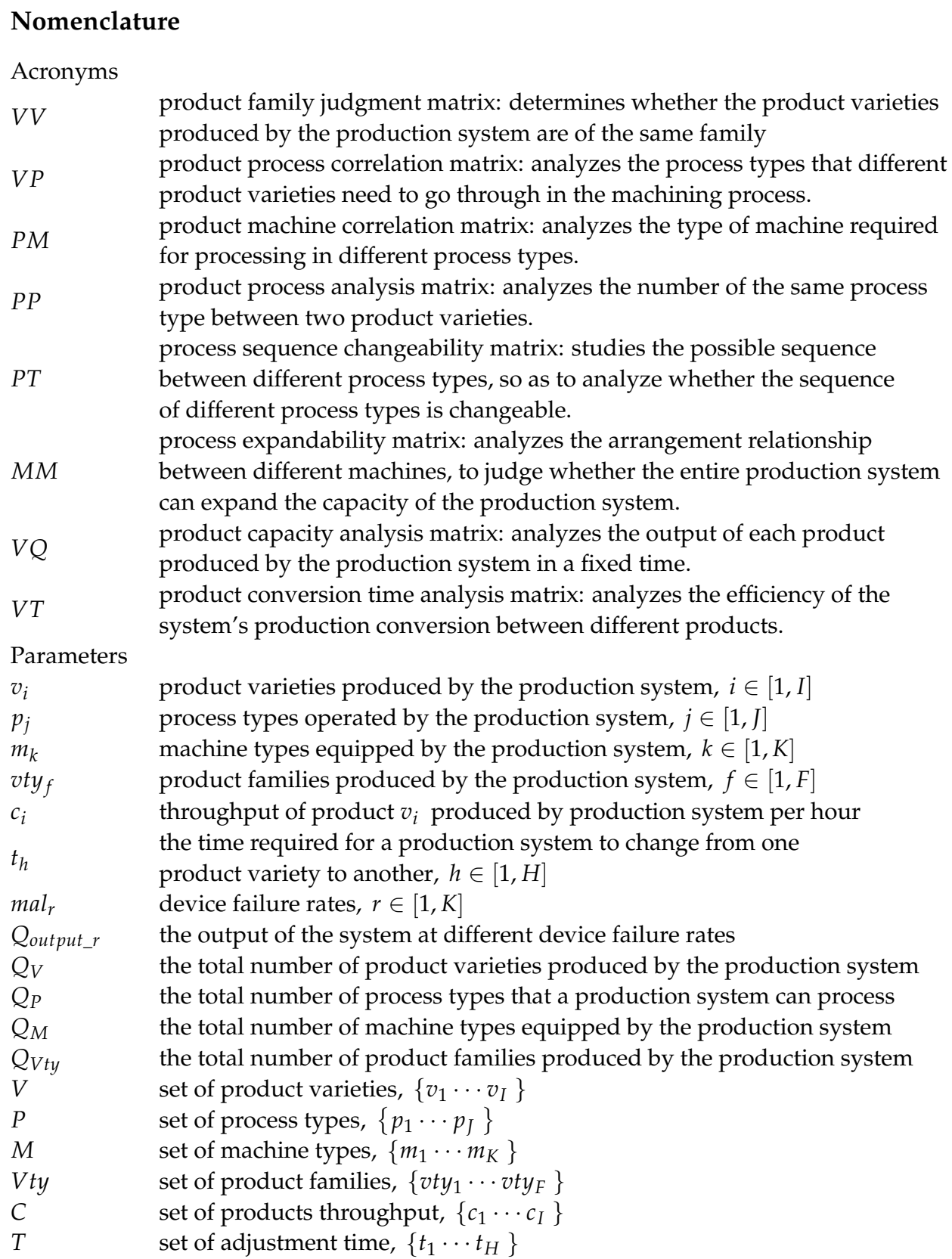

\section{References}

1. Zhang, L.L.; Lee, C.K.M.; Akhtar, P. Towards customization: Evaluation of integrated sales, product, and production configuration. Int. J. Prod. Econ. 2020, 229, 107775. [CrossRef]

2. Li, L. Research on discrete intelligent workshop lot-streaming scheduling with variable sublots under engineer to order. Comput. Ind. Eng. 2022, 165, 107928. [CrossRef]

3. Khalili-Araghi, S.; Kolarevic, B. Development of a framework for dimensional customization system: A novel method for customer participation. J. Build. Eng. 2016, 5, 231-238. [CrossRef]

4. Vinodh, S.; Devadasan, S.R.; Vimal, K.E.K.; Kumar, D. Design of agile supply chain assessment model and its case study in an Indian automotive components manufacturing organization. J. Manuf. Syst. 2013, 32, 620-631. [CrossRef]

5. Kim, J.; Choi, S.O. The Impact of Labor Flexibility on Innovation Performance at the Firm Level. Korean Manag. Rev. 2021, 50, 1435-1467. [CrossRef]

6. Marques, A.F.; Alves, A.C.; Sousa, J.P. An Approach for Integrated Design of Flexible Production Systems. Procedia CIRP 2013, 7 , 586-591. [CrossRef]

7. Lueder, A. Flexibility in Production Systems by Exploiting Cyberphysical Systems. Computer 2020, 53, 81-85. [CrossRef]

8. Sushil. Valuation of Flexibility Initiatives along the Value Chain. In Global Value Chains, Flexibility and Sustainability; Springer: Singapore, 2018; pp. 3-14. [CrossRef] 
9. Pérez, M.P.; Bedia, A.M.S.; López Fernández, M.C. A review of manufacturing flexibility: Systematising the concept. Int. J. Prod. Res. 2016, 54, 3133-3148. [CrossRef]

10. Sapol, S.J.; Szajnfarber, Z. Revisiting Flexibility in Design: An Analysis of the Impact of Implementation Uncertainty on the Value of Real Options. J. Mech. Des. 2020, 142, 121701-121712. [CrossRef]

11. Rogers, P.P.; Ojha, D.; White, R.E. Conceptualising complementarities in manufacturing flexibility: A comprehensive view. Int. J. Prod. Res. 2011, 49, 3767-3793. [CrossRef]

12. Nakajima, S.; Takisawa, J.; Ikari, S.; Tomooka, M.; Aoyanagi, Y.; Funase, R.; Nakasuka, S. Command-centric architecture (C2A): Satellite software architecture with a flexible reconfiguration capability. Acta Astronaut. 2020, 171, 208-214. [CrossRef]

13. Jahed, A.; Moghaddam, R.T. Mathematical Modeling for a Flexible Manufacturing Scheduling Problem in an Intelligent Transportation System. Iran. J. Manag. Stud. 2021, 14, 189-208. [CrossRef]

14. Jain, A.; Jain, P.K.; Chan, F.T.S.; Singh, S. A review on manufacturing flexibility. Int. J. Prod. Res. 2013, 51, 5946-5970. [CrossRef]

15. Liu, S.; Bao, J.; Lu, Y.; Li, J.; Lu, S.; Sun, X. Digital twin modeling method based on biomimicry for machining aerospace components. J. Manuf. Syst. 2021, 58, 180-195. [CrossRef]

16. Malik, A.A.; Brem, A. Digital twins for collaborative robots: A case study in human-robot interaction. Robot. Comput.-Integr. Manuf. 2021, 68, 102092. [CrossRef]

17. Lu, Y.; Liu, C.; Wang, K.I.K.; Wang, K.; Huang, H.; Xu, X. Digital Twin-driven smart manufacturing: Connotation, reference model, applications and research issues. Robot. Comput.-Integr. Manuf. 2020, 61, 101837. [CrossRef]

18. Chen, Z.; Huang, L. Digital twins for information-sharing in remanufacturing supply chain: A review. Energy 2021, $220,119712$. [CrossRef]

19. Kritzinger, W.; Karner, M.; Traar, G.; Henjes, J.; Sihn, W. Digital Twin in manufacturing: A categorical literature review and classification. IFAC-Pap. 2018, 51, 1016-1022. [CrossRef]

20. ISO/TC 184/SC 1 N517; Ad Hoc Group: Data Architecture of the Digital Twin. Ad Hoc Group, Inc.: Alexandria, VA, USA, 2019.

21. ISO 23247-1:2021; Automation Systems and Integration-Digital Twin Framework for Manufacturing-Part1: Overview and General Principles. International Organization for Standardization: Geneva, Switzerland, 2021. Available online: https://www. iso.org/standard/75066.html (accessed on 10 January 2022).

22. Ladj, A.; Wang, Z.; Meski, O.; Belkadi, F.; Ritou, M.; Da Cunha, C. A knowledge-based Digital Shadow for machining industry in a Digital Twin perspective. J. Manuf. Syst. 2021, 58, 168-179. [CrossRef]

23. Zhong, W.; Chen, J.; Zhou, Y.; Li, Z.; Lin, X. Network flexibility study of urban centralized heating system: Concept, modeling and evaluation. Energy 2019, 177, 334-346. [CrossRef]

24. Zheng, C.; Qin, X.; Eynard, B.; Bai, J.; Li, J.; Zhang, Y. SME-oriented flexible design approach for robotic manufacturing systems. J. Manuf. Syst. 2019, 53, 62-74. [CrossRef]

25. Zhang, Q.; Wu, D.; Fu, C.; Baron, C.; Peng, Z. A new method for measuring process flexibility of product design. Int. Trans. Oper. Res. 2017, 24, 821-838. [CrossRef]

26. Tolio, T.; Urgo, M. Design of flexible transfer lines: A case-based reconfiguration cost assessment. J. Manuf. Syst. 2013, 32, 325-334. [CrossRef]

27. Baykasoğlu, A. Quantifying machine flexibility. Int. J. Prod. Res. 2009, 47, 4109-4123. [CrossRef]

28. Oke, A. Linking manufacturing flexibility to innovation performance in manufacturing plants. Int. J. Prod. Econ. 2013, 143, 242-247. [CrossRef]

29. Vogel-Heuser, B.; Weber, J.; Folmer, J. Evaluating reconfiguration abilities of automated production systems in Industrie 4.0 with metrics. In Proceedings of the IEEE International Conference on Emerging Technologies and Factory Automation, Luxembourg, 8-11 September 2015.

30. Moral, C.; de Antonio, A.; Ferre, X.; Ramirez, J. A proposed UML-based common model for information visualization systems. Multimed. Tools Appl. 2021, 80, 12541-12579. [CrossRef]

31. Xu, X.; Chen, K.; Cai, H. Automating Utility Permitting within Highway Right-of-Way via a Generic UML/OCL Model and Natural Language Processing. J. Constr. Eng. Manag. 2020, 146, 04020135. [CrossRef]

32. Da Silva, A.R. Model-driven engineering: A survey supported by the unified conceptual model. Comput. Lang. Syst. Struct. 2015, 43, 139-155. [CrossRef]

33. Mamdikar, M.R.; Kumar, V.; Singh, P.; Singh, L. Reliability and performance analysis of safety-critical system using transformation of UML into state space models. Ann. Nucl. Energy 2020, 146, 107628. [CrossRef]

34. Vogel-Heuser, B.; Prieler, J. Evaluation of selected metrics for flexibility of Cyber Physical Production Systems. In Proceedings of the IEEE International Conference on Automation Science and Engineering, Xi'an, China, 20-23 August 2017. 